\title{
Associations Between Night Sleep Duration and Fasting Glucose and Triglyceride To High-Density Lipoprotein Cholesterol Ratio Among Adults Free of Type 2 Diabetes or Without Diagnosed Type 2 Diabetes
}

Jiangshan He

Nankai University

Chenglin Hong

University of California

Li Zhang

Tianjin First Central Hospital

Chunjun Li

Tianjin Union Medical Center

Yuxue Wang

Nankai University

Yaqi Fan

Nankai University

Pei Guo

Nankai University

Binbin Zhang

Nankai University

Xin Qi

Tianjin Union Medical Center

Shuo Chen

Beijing Physical Examination Center

Yujie Niu

Hebei Medical University

Feng Liu

Beijing Physical Examination Center

Rong Zhang

Hebei Medical University

Qiang Li

Beijing Physical Examination Center 


\section{Shitao Ma}

Hebei Medical University

\section{Mianzhi Zhang}

Tianjin Academy of Traditional Medicine Affiliated Hospital

Minying Zhang ( $\square$ zhangminying@nankai.edu.cn )

Nankai University

\section{Research Article}

Keywords: Sleep duration, Fasting glucose, Triglyceride to high-density lipoprotein cholesterol ratio, Body mass index

Posted Date: November 30th, 2021

DOI: https://doi.org/10.21203/rs.3.rs-1080358/v1

License: (c) (i) This work is licensed under a Creative Commons Attribution 4.0 International License. Read Full License 


\section{Abstract \\ Background}

We aim to assess the associations between night sleep duration and fasting glucose (FG), triglyceride (TG) to high-density lipoprotein cholesterol (HDL-C) ratio, and body mass index (BMI) among adults free of type 2 diabetes (T2D) or without diagnosed T2D.

\section{Methods}

We analyzed the baseline data of a cohort. We included adults free of T2D or without diagnosed T2D who completed the validated questionnaire, biochemical and anthropometric measurements.

Independent association between sleep duration and FG, TG/HDL-C, BMI was evaluated with multiple Ushaped or linear regressions.

\section{Results}

We included 32497 adults with a median of 44 years, $52.5 \%$ men. Overall, $12.80 \%$ and $9.67 \%$ reported night sleep duration $<7 \mathrm{~h}$ and $\geq 9 \mathrm{~h}, 6.91 \%$ were with impaired $\mathrm{FG}$ and $3.57 \%$ had undiagnosed T2D. Sleep duration was independently U-shaped associated with FG $\left[\beta_{1}\right.$ (linear term) $=-0.111, P=0.047 ; \beta_{2}$ (quadratic term) $=0.008, P=0.026]$ with 6.9-hour sleep had the lowest FG, and negatively associated with $\mathrm{BMI}(\beta=$ $-0.154, P<0.001)$. BMI mediated U-shaped association of sleep duration with TG/HDL-C $\left(\beta_{1}=-0.040\right.$, $P=0.017 ; \beta_{2}=0.003, P=0.023$.

\section{Conclusions}

Both short and long night sleep was associated with elevated FG, shorter sleep was associated with increased BMI. BMI mediated the U-shaped association between sleep duration and TG/HDL-C.

\section{Introduction}

According to the International Diabetes Federation Diabetes ATLAS (9th edition) data released in 2019, approximately 463 million adults aged 20-79 were suffering from diabetes globally, with a crude prevalence rate of $9.3 \%[1]$. Type 2 diabetes (T2D), accounting for around $90 \%$ of all diabetes worldwide, has shown an increased prevalence in China in recent decades. In parallel, there has been an increased sleep deprivation in China[2]. Sleep is a fundamental and complex physiological process. Long-term sleep deprivation will cause a series of physical and mental illnesses[3]. Optimal sleep duration is one of the critical factors to maintain metabolic balance[4]. Both short and long sleep duration reduce insulin sensitivity and induce the production of a variety of inflammatory cytokines, which will affect the body's 
glucose and lipid metabolism and induce or accelerate the occurrence and development of metabolic diseases such as obesity, metabolic syndrome, and T2D[5]. Besides, sleep deprivation can also cause physiological and behavioral changes, such as an increase in wakefulness that leads to increased energy consumption, which leads to an unbalanced increase in food intake, reduction in physical activity, and overweight, thus further increases T2D risk[6].

In general, T2D may be completely symptomless, and the exact time of the onset of T2D is usually impossible to determine. As a result, there is often a long pre-diagnostic period, and as many as one-third to one-half of people with T2D in the population may be undiagnosed[1]. T2D is characterized by insulin resistance, which occurs before the clinical diagnosis of T2D[7]. A substantial number of individuals free of T2D or without diagnosed T2D are suffering from insulin resistance and will be later diagnosed with diabetes. Consequently, early identification of insulin resistance in these high-risk groups is essential in preventing T2D.

Although there has mounting evidence that short or long sleep duration is related to the risk of T2D and the control of fasting glucose (FG) among patients with T2D $[8,9]$, only a few have examined the association between sleep duration and predictors of insulin resistance, FG and Triglycerides (TG) to high-density lipoprotein cholesterol (HDL-C) ratio (TG/HDL-C) $[10,11]$, and the results were equivocal[12, 13]. As such, this current study aims to explore the associations of self-reported night sleep duration and FG, TG/HDL-C, and body mass index (BMI) among people free of T2D or without diagnosed T2D, which will provide evidence to sensitively and directly identify the impact of sleep duration on insulin resistance.

\section{Methods}

\section{Study Design and Recruitment}

We conducted a cross-sectional study among adults for health screening using cluster sampling from January 2018 to January 2020 in the Beijing-Tianjin-Hebei Region, China. To be eligible for the study, participants were required to (1) aged 18 years and above; (2) agreed to participate in the study and signed the informed consent. Individuals were excluded from this analysis if they (1) were with cognitive impairment, hearing impairment, articulate problems, or severe mental illness that cannot complete the survey; (2) were in acute infection status, such as fever, diarrhea, etc.; (3) had been diagnosed with type 1 or type 2 diabetes and were taking hypoglycemic drugs or injecting insulin; (4) had been diagnosed with hyperlipidemia and were taking lipid-lowering drugs; (5) were with missing one of the measurements of FG, TG, HDL-C, height, and weight. The study protocol was approved by the ethics review boards of Nankai University (NKUIRB2016063), Tianjin First Central Hospital (2017N052KY), Tianjin Union medical center (2018C02) and Hebei Medical University (2016021). The research procedures were carried out strictly following the Declaration of Helsinki. All methods were carried out in accordance with relevant guidelines and regulations. Written informed consent was obtained from each participant.

\section{Data Collection and Measurement}




\section{Sleep Assessments}

The modified Pittsburgh Sleep Quality Index (PSQI) questionnaire, which has been proved to have high reliability and validity in Chinese population[14], was used to determine self-reported items. Participants were asked questions regarding sleep in the past month: "when have you usually gone to bed at night?", "when have you usually gotten up in the morning?" and "how long does it usually take you to fall asleep each night?". We calculated participants' average daily night sleep duration based on these questions. The average self-reported sleep duration was reported by hours and was calculated by the time to fall asleep and the time to get up. If they did not indicate an exact period, we used the filled period's middle time point. For example, if participants report they usually go to bed at 22:00 23:00, then we used 22:30 as the point of time. According to the distribution of different night sleep duration and studies[15], the self-reported sleep duration was analyzed categorically ( $<7$ hours, 7 to $<8$ hours, 8 to $<9$ hours, and $\geq 9$ hours). Subjective sleep quality was asked by the following question: "What do you think of your sleep quality in the prior month?". Answer options included excellent, good, bad, and awful. Subjective sleep quality has been proved to have the highest correlation with the global score among the seven factors when researchers evaluated the Chinese version of PSQI[16], thus we used it to assess the participants' sleep quality. Besides, participants were also asked, "How often do you work at night shift in the past month?" and "how often do you use sleeping medication?". Response options included (1) not during the past month; (2) less than once a week; (3) once or twice a week; (4) three times or more a week.

\section{Fasting glucose, Triglycerides, and High-Density Lipoprotein Cholesterol}

Participants were required to fast for at least 12 hours before the blood sample was obtained the following morning. TC and HDL-C were measured using the Hitachi 7600 automated biochemical analyzer (Hitachi, Inc., Tokyo, Japan). All procedures were performed by the trained professional medical assistant and staff at the medical examination centers.

\section{Anthropometric measurement}

All anthropometric measurements were assessed with participants wearing light clothing without shoes before breakfast. Height was measured to the nearest $0.1(\mathrm{~cm})$, and weight $(\mathrm{kg})$ was measured to the nearest $0.1(\mathrm{~kg})$ by the professional medical staff of the medical examination centers using the same calibrated electronic scale (GL-310, Seoul, Korea), zeroed before each measurement. BMI was expressed as body weight $(\mathrm{kg})$ divided by squared body height $\left(\mathrm{m}^{2}\right)$.

\section{Demographic Characteristics}

Participants were asked to report their age, gender, ethnic group, highest education level, marital status, and employment status.

\section{Other Covariates}


Alcohol drinking, smoking and physical exercise were also evaluated in the questionnaire. Participants who reported had been smoking for more than half a year were defined as smokers. In contrast, those who reported having smoked but later quit for a sustained period of half a year or longer by the interview time were former smokers. Those who indicated consuming alcohol at least once a week were defined as alcohol drinkers. Those that had been away from drinking alcohol for half a year or longer by the time of the interview were drinking abstainers. Physical exercise was described as an exercise once a week or more for at least half an hour each time.

\section{Statistical Analysis}

Data were input, stored, and managed centrally. Sleep duration was categorized into 4 groups $(<7 \mathrm{~h}, 7$ to $<8 \mathrm{~h}, 8$ to $<9 \mathrm{~h}$, and $\geq 9 \mathrm{~h}$ ) in the statistical analyses. Outcomes in this study were FG, TG/HDL-C, and BMI. Continuous variables tested normally distributed were expressed as mean \pm SD. TG/HDL-C, which conformed to a right-skewed distribution, was logarithmically converted to lg (TG/HDL-C). Oneway analysis of variance (ANOVA) was used to perform the unadjusted group comparisons of normally distributed continuous variables. The other continuous variables not normally distributed were summarized as median with interquartile range (IQR). Categorical variables were described as percentages and compared using Pearson's test.

Based on the scatter plot and the mean graph of ANOVA, we assumed that night sleep duration has curvilinear (U-shaped) correlations with FG and TG/HDL-C and linear correlation with BMI. Multiple linear and curvilinear regression models were performed to identify the assumed independent linear or Ushaped associations of FG, TG/HDL-C, and BMI with sleep duration using FG and TG/HDL-C as dependent variables. In Model 1, FG and TG/HDL-C were regressed on night sleep duration after adjusting for sex, age, occupation, highest education, marriage status, physical exercise, BMI, subjective sleep quality, and sleeping drug use. In Model 2, FG and TG/HDL-C were regressed on night sleep duration using both a linear and a quadratic term to identify the assumed U-shaped relation after adjusting for sex and age. In Model 3, BMI was additionally included as a possible explaining factor to the relation tested in Model 2. In Model 4, occupation, highest education, marriage status, physical exercise, subjective sleep quality, and sleeping drug use were additionally included as possible confounders.

In Model 5, a multiple linear regression model was performed to explore the independent association of BMI with sleep duration after adjustment of sex, age. In Model 6, occupation, highest education, marriage status, physical exercise, subjective sleep quality, and sleeping drug use were included as potential confounding factors. It was tested whether the linear associations of BMI with sleep duration in Model 5 remain present. Two-side $P<0.05$ was considered statistically significant. We used Epidata 3.0 to input and manage the data and Stata 15.1 (College Station, TX, USA) to analyze the data.

\section{Results}




\section{Participants' Characteristics}

Overall, 40,525 participants completed the baseline questionnaire. After excluding those who were currently taking hypoglycemic agents $(n=2,322)$, or lipid-lowering agents $(n=780)$, or missing metabolic indicators (FG, TG, HDL-C), or height or weight $(n=4,944)$, a total of 32,497 individuals were included in the final analysis. The study population selection process is shown in Figure 1. Key participant's characteristics are summarized in Table 1. Participants' age ranged from 18 to 99 (median = 44, IQR: 3556) years, and over half (52.5\%) of the participants were men. Self-reported sleep duration and sleep quality were also presented in Table 1 . Specifically, characteristics were stratified by sleep duration. In short, the proportions of participants who reported different sleep durations were $12.80 \%$ ( $<7$ hours), $38.06 \%$ ( 7 to $<8$ hours), $39.47 \%$ ( 8 to $<9$ hours), and $9.67 \%$ ( $\geq 9$ hours). More than forty percent ( $42.54 \%$ ) of the participants reported excellent sleep quality. None of the participants work on the night shift in our cohort. 
Table 1

Characteristics of participants without diagnosed diabetes according to level of night sleep duration

\begin{tabular}{|c|c|c|c|c|c|}
\hline \multirow[t]{2}{*}{ Characteristic } & \multicolumn{4}{|c|}{ Night sleep duration (h) } & \multirow[t]{2}{*}{$P$} \\
\hline & $<7$ & $7<8$ & $8<9$ & $\geq 9$ & \\
\hline Age category (\%) & & & & & $<0.001$ \\
\hline $18-30$ years & $294(7.1)$ & $1258(10.2)$ & $1431(11.2)$ & $411(13.1)$ & \\
\hline $31-40$ years & $1047(25.2)$ & $3644(29.5)$ & $4236(33.0)$ & $852(27.1)$ & \\
\hline $41-50$ years & $1176(28.3)$ & $3185(25.7)$ & $2617(20.4)$ & $387(12.3)$ & \\
\hline $51-60$ years & $893(21.5)$ & $2276(18.4)$ & $2303(18.0)$ & $641(20.4)$ & \\
\hline $61-70$ years & $528(12.7)$ & $1318(10.7)$ & $1388(10.8)$ & $446(14.2)$ & \\
\hline 71-99 years & $221(5.3)$ & 689 (5.6) & $850(6.6)$ & $406(12.9)$ & \\
\hline $\operatorname{Sex}(\%)$ & & & & & $<0.001$ \\
\hline Men & $2519(60.6)$ & $6828(55.2)$ & $6246(48.7)$ & $1453(46.2)$ & \\
\hline Women & $1640(39.4)$ & $5542(44.8)$ & $6579(51.3)$ & $1690(53.8)$ & \\
\hline Ethnicity (\%) & & & & & 0.133 \\
\hline Han & $3963(95.3)$ & $11849(95.8)$ & $12326(96.1)$ & $3013(95.9)$ & \\
\hline Others & $196(4.7)$ & $521(4.2)$ & 499 (3.9) & $130(4.1)$ & \\
\hline Marital status (\%) & & & & & $<0.001$ \\
\hline Unmarried & $402(9.7)$ & $1624(13.1)$ & $1661(13.0)$ & $414(13.2)$ & \\
\hline Married & $3662(88.0)$ & $10528(85.1)$ & 10963 (85.5) & $2644(84.1)$ & \\
\hline Divorced & $61(1.5)$ & $134(1.1)$ & $120(0.9)$ & $34(1.1)$ & \\
\hline Widowed & $34(0.8)$ & $84(0.7)$ & $81(0.6)$ & $51(1.6)$ & \\
\hline Education (\%) & & & & & $<0.001$ \\
\hline Primary school & $82(2.0)$ & $136(1.1)$ & $167(1.3)$ & $73(2.3)$ & \\
\hline Junior high school & $386(9.3)$ & 909 (7.3) & $813(6.3)$ & $270(8.6)$ & \\
\hline Senior high school & $617(14.8)$ & $1593(12.9)$ & $1577(12.3)$ & $472(15.0)$ & \\
\hline Undergraduate & $2412(58.0)$ & 7348 (59.4) & 7598 (59.2) & $1766(56.2)$ & \\
\hline
\end{tabular}

Data are $\mathrm{n}(\%)$ or mean $\pm \mathrm{SD}$. $P$ value comparing the four groups of night sleep duration.

a TG/HDL-C levels are presented as Geometric mean (-1SD, +1SD). 


\begin{tabular}{|c|c|c|c|c|c|}
\hline \multirow[t]{2}{*}{ Characteristic } & \multicolumn{4}{|c|}{ Night sleep duration (h) } & \multirow[t]{2}{*}{$P$} \\
\hline & $<7$ & $7<8$ & $8<9$ & $\geq 9$ & \\
\hline Graduate or above & $662(15.9)$ & $2384(19.3)$ & $2670(20.8)$ & $562(17.9)$ & \\
\hline Occupation (\%) & & & & & $<0.001$ \\
\hline Workers & 485 (11.7) & $1412(11.4)$ & $1479(11.5)$ & $380(12.1)$ & \\
\hline Civil servants & $1184(28.5)$ & $3684(29.8)$ & $3810(29.7)$ & $853(27.1)$ & \\
\hline $\begin{array}{l}\text { Service sales } \\
\text { staffs }\end{array}$ & $592(14.2)$ & $1235(10.0)$ & $1010(7.9)$ & $225(7.2)$ & \\
\hline Professionals & $1553(37.3)$ & $4920(39.8)$ & $5130(40.0)$ & $1215(38.6)$ & \\
\hline Others & $345(8.3)$ & $1119(9.0)$ & $1396(10.9)$ & $470(15.0)$ & \\
\hline $\begin{array}{l}\text { Physical exercise } \\
(\%)\end{array}$ & & & & & $<0.001$ \\
\hline Never & $1539(37.0)$ & $3928(31.8)$ & $4010(31.3)$ & $1071(34.1)$ & \\
\hline Occasionally & $1303(31.3)$ & $4196(33.9)$ & $4536(35.4)$ & $1062(33.8)$ & \\
\hline Regularly & $1317(31.7)$ & $4246(34.3)$ & $4279(33.3)$ & $1010(32.1)$ & \\
\hline \multicolumn{5}{|c|}{ Subjective sleep quality (\%) } & $<0.001$ \\
\hline Excellent & $1632(39.2)$ & $5203(42.1)$ & $5562(43.4)$ & $1426(45.4)$ & \\
\hline Good & $1820(43.8)$ & $5699(46.1)$ & $6037(47.1)$ & $1402(44.6)$ & \\
\hline Bad & $631(15.2)$ & $1353(10.9)$ & $1122(8.7)$ & $291(9.3)$ & \\
\hline Awful & $76(1.8)$ & $115(0.9)$ & $104(0.8)$ & $24(0.8)$ & \\
\hline \multicolumn{5}{|c|}{ Taking sleep medications (\%) } & $<0.001$ \\
\hline Never & $3972(95.5)$ & $12028(97.2)$ & $12499(97.4)$ & $3027(96.3)$ & \\
\hline Once/week & $66(1.6)$ & $130(1.1)$ & $138(1.1)$ & $39(1.3)$ & \\
\hline $\begin{array}{l}\text { Once or twice a } \\
\text { week }\end{array}$ & $51(1.2)$ & $90(0.7)$ & $86(0.7)$ & $29(0.9)$ & \\
\hline$\geq 3$ times/week & $70(1.7)$ & $122(1.0)$ & $102(0.8)$ & $48(1.5)$ & \\
\hline $\mathrm{FG}(\mathrm{mmol} / \mathrm{L})$ & $5.32 \pm 0.99$ & $5.26 \pm 0.95$ & $5.26 \pm 0.99$ & $5.33 \pm 1.10$ & $<0.001$ \\
\hline $\mathrm{BMI}\left(\mathrm{kg} / \mathrm{m}^{2}\right)$ & $25.00 \pm 3.78$ & $24.56 \pm 3.73$ & $24.23 \pm 3.64$ & $24.15 \pm 3.61$ & $<0.001$ \\
\hline
\end{tabular}

Data are $\mathrm{n}(\%)$ or mean $\pm \mathrm{SD}$. $P$ value comparing the four groups of night sleep duration.

a TG/HDL-C levels are presented as Geometric mean (-1SD, +1SD). 


\begin{tabular}{|llllll|}
\hline Characteristic & \multicolumn{4}{l}{ Night sleep duration (h) } & $P$ \\
\cline { 2 - 6 } & $<7$ & $\mathbf{7}<8$ & $\mathbf{8}<9$ & $\geq 9$ & \\
\hline TG $(\mathrm{mmol} / \mathrm{L})$ & $1.57 \pm 1.30$ & $1.50 \pm 1.20$ & $1.45 \pm 1.12$ & $1.48 \pm 1.23$ & $<0.001$ \\
\hline HDL-C (mmol/L) & $1.32 \pm 0.32$ & $1.33 \pm 0.32$ & $1.34 \pm 0.32$ & $1.34 \pm 0.32$ & 0.001 \\
\hline TG/HDL-C & $1.01(0.50$, & $0.96(0.48$, & $0.93(0.47$, & $0.95(0.48$, & $<0.001$ \\
\hline Sample size (\%) & $2.05)$ & $1.94)$ & $1.86)$ & $1.89)$ & \\
\hline Data are n (\%) or mean \pm SD. P value comparing the four groups of night sleep duration. & \\
\hline a TG/HDL-C levels are presented as Geometric mean (-1SD, +1SD). & & \\
\hline
\end{tabular}

Nearly ninety percent of participants (89.52\%) had normal blood glucose ( $\leq 6.0 \mathrm{mmol} / \mathrm{L}), 6.91 \%$ had impaired FG, and 3.57\% had T2D but were not diagnosed or received any treatment. The averages of FG, BMI, TG, HDL-C and TG/HDL-C were $5.28 \mathrm{mmol} / \mathrm{L}, 24.45 \mathrm{~kg} / \mathrm{m}^{2}, 1.49 \mathrm{mmol} / \mathrm{L}, 1.33 \mathrm{mmol} / \mathrm{L}$, and 0.96, respectively. The association between age, gender, marital status, education level, employment status, physical exercise, subjective sleep quality, and the use of sleeping medication, FG, BMI, TG, HDL-C, TG/HDL-C were all statistically significantly different in sleep duration (all $P<0.001$ ). Ethnicity was not significantly associated with varying durations of sleep.

\section{Associations of Night Sleep Duration with FG, TG/HDL-C, and BMI}

Results of one-way ANOVA were presented in Table 2. We found statistically significant differences in FG, TG/HDL-C and BMI at different night sleep durations (all $P<0.001$, shown in Figure 2). Compared with those reported sleep durations was 7 to $<8$ hours, FG was higher among sleep duration was $<7$ hours and $\geq 9$ hours ( $P=0.018$ and $P=0.008$, respectively). Similarly, TG/HDL-C was significantly higher among sleep duration $<7$ hours and 8 to $<9$ hours than the reference group ( $P=0.001$ and $P=0.002$, respectively). In terms of BMI, the average BMI among those who reported less than 7 hours of sleep duration was significantly higher than the other three groups ( $P \leq 0.001$ for all).

We observed a U-Shaped relationship for sleep duration and FG and therefore tested whether the Ushaped relationship was statistically significant. Results were shown in Table 2. In the completely adjusted multiple linear regression Model 1 (adjusted for sex, age, BMI, occupation, marital status, education, physical exercise, subjective sleep quality, taking sleep medications), night sleep duration is positively associated with FG $(B=0.012, P=0.029)$. In multiple curvilinear regression Model 2 (adjusted for age and sex), both the linear and the quadratic terms of night sleep duration are statistically significant $\left(\mathrm{B}_{1}=-0.153, P=0.007 ; \mathrm{B}_{2}=0.011, P=0.004\right)$. The $\mathrm{U}$-shaped relationship remains present in Model 3 where we adjusted for age, sex, and BMI. In the multiple curvilinear regression model (Model 4) completely adjusted for sex, age, BMl, occupation, marital status, education, physical exercise, subjective sleep 
quality and taking sleep medications, both the linear and the quadratic term of night sleep duration are statistically significant $\left(B_{1}=-0.111, P=0.047 ; B_{2}=0.008, P=0.026\right)$, indicating the U-shaped relationship between night sleep duration and FG, and the top point calculated is 6.9 hours.

We used the same statistical models to analyze the association between night sleep duration and TG/HDL-C. Specifically, night sleep duration is not significantly associated with $\lg (T G / H D L-C)$ in the completely adjusted linear regression Model 1 (adjusted for sex, age, BMl, occupation, marital status, education, physical exercise, subjective sleep quality, and taking sleep medications, $P=0.304)$. After adjusting for sex and age in curvilinear regression model (Model 2), both the linear and the quadratic terms of night sleep duration are significant $\left(B_{1}=-0.040, P=0.017 ; B_{2}=0.003, P=0.023\right)$. However, in Model 3 when we adjusted for sex, age, and BMI, neither the linear nor the quadratic term of night sleep duration was significant anymore $(P=0.139, P=0.117)$. In the final curvilinear regression model (Model 4) completely adjusted for sex, age, BMI, occupation, marital status, education, physical exercise, subjective sleep quality, taking sleep medications, neither the linear nor the quadratic term of night sleep duration was statistically significant $(P=0.365, P=0.310)$.

Lastly, the BMI changes in different sleep duration categories showed a linear tendency, so we established multiple linear regression models to test whether the association is statistically significant. In the model adjusted for age and sex (Model 5), night sleep duration was inversely associated with BMI $(B=-0.154, P<0.001)$. In Model 6, which was completely adjusted for sex, age, occupation, marital status, education, physical exercise, subjective sleep quality, taking sleep medications, BMI remains independently associated with night sleep duration $(B=-0.154, P<0.001)$. For each hour of additional night sleep duration, the adjusted BMI decreased by $0.15 \mathrm{~kg} / \mathrm{m}^{2}$. 
Table 2

Multiple linear regression results of $\mathrm{FG}$ and $\mathrm{Ig}(\mathrm{TG} / \mathrm{HDL}-\mathrm{C})$ according to night sleep duration

\begin{tabular}{|llll|}
\hline & Sleep duration & $\mathbf{B}(95 \% \mathrm{Cl})$ & $\boldsymbol{P}$ \\
\hline FG & & & \\
\hline Model 1 & linear term & $0.012(0.001,0.024)$ & 0.029 \\
\hline Model 2 & linear term & $-0.153(-0.263,-0.042)$ & 0.007 \\
\hline Model 3 & quadratic term & $0.011(0.003,0.018)$ & 0.004 \\
\hline Model 4 & linear term & $-0.126(-0.236,-0.017)$ & 0.024 \\
\hline lg (TG/HDL-C) & quadratic term & $0.009(0.002,0.017)$ & 0.012 \\
\hline Model 1 & linear term & $-0.111(-0.221,-0.002)$ & 0.047 \\
\hline Model 2 & linear term & $-0.040(-0.073,-0.007)$ & 0.017 \\
\hline Model 3 & quadratic term & $0.003(0.000,0.005)$ & 0.023 \\
\hline linear term & $-0.024(-0.056,0.008)$ & 0.139 \\
\hline Model 4 & quadratic term & $0.002(0.000,0.004)$ & 0.117 \\
\hline Model 5 & linear term & $-0.015(-0.046,0.017)$ & 0.365 \\
\hline quadel 6 & linear term & $-0.154(-0.195,-0.114)$ & $<0.001$ \\
\hline
\end{tabular}

Model 1: Completely adjusted multiple linear regression model (adjusted for sex, age, body mass index, occupation, marital status, education, physical exercise, subjective sleep quality, taking sleep medications)

Model 2: U-shaped test regression model (adjusted for sex, age)

Model 3: U-shaped test regression model (adjusted for sex, age, body mass index)

Model 4: U-shaped test regression model (adjusted for sex, age, body mass index, occupation, marital status, education, physical exercise, subjective sleep quality, taking sleep medications) 
Model 5: Multiple linear regression model (adjusted for sex, age)

Model 6: Multiple linear regression model (adjusted for sex, age, occupation, marital status, education, physical exercise, subjective sleep quality, taking sleep medications)

\section{Discussions}

Using a large, FG diverse sample free of T2D or without diagnosed and untreated T2D, our current study demonstrated an independent U-shaped relationship between sleep duration and FG with the optimal sleep duration 6.9 hours, indicating that both short and long night sleep were associated with higher measures of glycemia after controlling for demographic characteristics, lifestyles and BMI. We also found a BMI mediated association between sleep duration and TG/HDL-C after adjusting for sex and age, and an inverse linear association of sleep duration with BMI after control of demographic characteristics and lifestyles.

The findings of the U-shaped relationship between sleep duration and FG are consistent with the research among overweight or obese adults with prediabetes or recently diagnosed untreated T2D in the United States[12]. Similar results were also found in a cross-sectional study among adults aged over 40 in China[17], but the association was only found among female participants, where short ( $<6$ hours) and long ( $\geq 8$ hours) night sleep duration increased the risk of higher measures of glycemia $(O R=1.12$, $\mathrm{OR}=1.14$ ). However, findings on the relationship between sleep duration and glycemia or diabetes were equivocal. A cross-sectional study conducted in South Korea only observed the association between short sleep duration and impaired fasting glucose (IFG) among male participants[18], whereas long sleep duration was not associated with IFG. However, a cohort study conducted in China among middle-aged and older adults revealed that long sleep duration was an independent predictor of incident diabetes[8], and similar results were confirmed among a group of retired Chinese adults[9]. We speculate there are several potential reasons for the inconsistency of the results. First, the above studies were conducted in different settings and among diverse populations, where sleep duration and subjective sleep quality vary across cultures and countries[19,20], thus may alter the associations between sleep profiles and diabetes risks[21, 22]. Second, differences in participants' demographic characteristics may also influence the correlation between sleep duration and blood glucose.

Multiple experimental studies have assessed the role of sleep in controlling energy balance and glucose metabolism and revealed that sleep restrictions could lead to glucose dysregulation through several biological pathways. One is by altering levels of neurohormones that regulate eating behaviors. It is suggested that acute sleep deprivation leads to a $28 \%$ increase in the average level of appetitestimulating hormones, while the average level of leptin decreases by $18 \%$. Besides, lack of sleep is associated with increases in hunger, appetite, and hedonic food intake, especially for high-calorie food[23]. Another possible way for insufficient sleep to induce T2D is through activation of the sympathetic nervous system. Insufficient sleep can reduce glucose tolerance, increase cortisol levels and heart rate variability. These neuroendocrine changes interfere with the mechanism of regulating blood 
glucose, resulting in elevated steady-state blood glucose concentrations[24]. Additionally, activation of inflammatory pathways may also play a role in the link between short sleep duration and impaired glucose metabolism. Studies have confirmed that insufficient sleep is associated with increases in tumor necrosis factor, interleukin 6 and C-reactive protein[25, 26]. Long sleep duration has many similar potential metabolic problems, but it may differ from short sleep duration[6, 27]. Several studies have demonstrated that prolonged sleep duration can affect energy metabolism throughout the human body and thereby increase the risk of T2D through various possible complex mechanisms, including poor sleep quality, sedentary lifestyle, unhealthy dietary habits, and circadian rhythm[28]. These results suggest that interventions in behavioral patterns such as a sedentary lifestyle may promote healthy sleep habits, balance fasting blood glucose, thus prevent T2D.

In the current study, we only observed the U-shape relationship between night sleep duration and TG/HDL$\mathrm{C}$ after adjusting for age and gender. After adjusting for $\mathrm{BMI}$, this association was no longer significant, suggesting BMI may mediate the association between sleep duration and TG/HDL-C. At present, the association between sleep duration and TG/HDL-C is not consistent in the literature. A cross-sectional study of Danish adults did not find a significant correlation between sleep duration and TG/HDL-C[29], while a cohort study among Mexican adolescents revealed that the two variables were independently related[30]. Considering the importance of adjusting BMI on the results, there are at least two explanations for the BMI adjusted model. First, insufficient sleep and sleep disturbance may increase $\mathrm{BMI}$, leading to impaired glucose homeostasis. There is evidence that BMI may mediate the association between insulin and glycosylated hemoglobin concentrations and short sleep duration[31]. Secondly, our research found that BMI decreases with the increase of sleep duration, and thereby BMI may be a confounding variable between sleep duration and TG/HDL-C[31]. Future studies should research the effect of sleep duration on insulin resistance.

Lastly, our findings revealed an inverse association between night sleep duration and BMI. This finding is consistent with previous studies among adolescents and adults[32, 33], and the association was generally consistent across different categories of age, educational level, smoking status, baseline body mass index, and physical activity level[34]. Although some studies suggested that long sleep duration is also a risk factor for obesity and BMI increase[35,36], the evidence is insufficient among different races and populations[37]. Many factors such as race/ethnicity, tobacco use, sedentary behaviors may confound the proposed association[34]. Short sleep duration has been proved to be a potential risk factor for obesity and insulin resistance[5]. Insulin resistance is considered a common pathological basis of metabolic syndrome and a risk factor for T2D[38]. This study found that sleep duration is U-shaped with blood glucose, linearly related to BMI, and the U-shaped relationship with TG/HDL-C disappeared after adjusting BMI, which suggested that sleep duration has more complex effects on blood glucose and insulin resistance. These associations may also indicate that short sleep duration can either directly increase blood glucose or affect insulin resistance through different pathways, including obesity, which indirectly leads to glucose dysregulation and breaks blood glucose homeostasis. It can also directly increase insulin resistance by changing glucose metabolism[39]. Our findings suggest that an increase 
within a reasonable range in sleep duration can reduce excessive fasting blood glucose and reduce BMI simultaneously, thereby reducing the risk of T2D.

Our study has several strengths. First, our cohort has a large number of adults as study samples. The age and gender composition are close to that of the total Chinese population[40], the age span is large (18-99 years), and participants have diverse occupational and educational backgrounds. All these increased the generalizability of our findings. Secondly, we studied adults with various diabetes status, including adults with normal blood glucose, prediabetes (impaired blood glucose), and adults with T2D but were not diagnosed or receiving treatment. In addition, we excluded adults taking lipid-lowering drugs. Therefore, the interference of treatment on blood glucose and blood lipids was well controlled when analyzing the effect of sleep on FG and TG/HDL-C. Lastly, this is a multi-site study. Participants were recruited from 7 different centers in the Beijing-Tianjin-Hebei Region, and a standardized sleep questionnaire was used across all centers. All metabolic markers' measurements adopted the same methods and standards, which substantially reduced the measurement errors and improved the results' reliability.

The strengths should be interpreted with the limitations. Due to this study's cross-sectional nature, we cannot examine the temporality of the associations between sleep duration and FG, TG/HDL-C, and BMI. Meanwhile, sleep duration and sleep quality were self-reported and subject to social desirability bias and recall bias. Future research should implement objective measurements to obtain more accurate sleep durations. However, a study showed good correlations between actigraphy and sleep logs when measuring sleep duration, and the self-reported data were reliable[41]. It is not feasible to obtain sleep duration through actigraphy due to the large sample size of this study. Therefore, we believe self-reported data collection was the most appropriate approach.

\section{Conclusion}

There is an independent U-shaped association between night sleep duration and FG with the optimal sleep duration 6.9 hours, indicating that both short and long sleep are associated with elevated blood glucose levels. The inverse linear association of sleep duration and BMI indicates short sleep is associated with elevated BMI while long sleep is associated with decreased BMI. In addition, the Ushaped relationship between sleep duration and TG/HDL-C is mediated by BMI. It is suggested that sleep affects blood glucose and insulin resistance through various mechanisms, and one of them may be through BMI. Prospective research is warranted to better delineate the relationship between night sleep duration and glycemia. Our findings suggest that optimal sleep duration is important for maintaining glucose homeostasis, individuals with impaired FG or undiagnosed T2D may require more clinical attention targeting sleep duration to decrease the conversion from prediabetes to diabetes or delay the progression of diabetes.

\section{Abbreviations}


BMI: body mass index; FG: Fasting Glucose; HDL-C: High-Density Lipoprotein Cholesterol; PSQI: Pittsburgh Sleep Quality Index; T2D: Type 2 Diabetes; TG: Triglyceride

\section{Declarations}

\section{Ethics approval and consent to participate}

The study protocol was approved by the ethics review boards of Nankai University (NKUIRB2016063), Tianjin First Central Hospital (2017N052KY), Tianjin Union medical center (2018C02) and Hebei Medical University (2016021). The research procedures were carried out strictly following the Declaration of Helsinki. All methods were carried out in accordance with relevant guidelines and regulations. Written informed consent was obtained from each participant.

\section{Consent for publication}

Not applicable.

\section{Availability of data and materials}

The data that support the findings of this study are available on request from the corresponding author My Z. The data are not publicly available due to state restrictions them containing information that could compromise research participant privacy.

\section{Competing interests}

The authors declare that they have no competing interests.

\section{Funding}

This work was supported by the National Key Research and Development Program of China (2016YFC0900600, 2016YFC0900604). The funders had no involvement in or influence on this study.

\section{Authors' contributions}

All authors have contributed substantially to the study. My Z. has designed the study, supervised and oversaw the study implementation, and wrote the manuscript. J.H. conducted the investigation and analysis of the data. J.H. and C.H. wrote the manuscript. L.Z., C.L., S.C., X.Q., Y.N. and Mz.Z. have organized and managed the investigation. B.Z., Y.W., Y.F, P.G., F.L., R.Z., Q.L., S.M., L.Z., C.L., S.C., X.Q., Y.N. and Mz.Z. have participated in the investigation and the management of the data. All authors read and approved the final manuscript. My.Z. is the guarantors of this work and, as such, had full access to all of the data in the study and take responsibility for the integrity of the data and the accuracy of the data analysis.

\section{Acknowledgements}


The survey was performed in Tianjin Union Medical Center, Tianjin First Central Hospital, Beijing Physical Examination Center, the First Hospital of Hebei Medical University, the Second Hospital of Hebei Medical University, and Hebei Changcheng Health Screening Center. We sincerely thank all the study participants and the staff involved in facilitating and running the study.

\section{References}

1. IDF DIABETES ATLAS 9th edition 2019. Available from: https://www.diabetesatlas.org/en/. Accessed 23 January 2021

2. Deng, H. B. et al. Short Sleep Duration Increases Metabolic Impact in Healthy Adults: A PopulationBased Cohort Study. Sleep 2017;40.

3. Morin, C. M. \& Benca, R. Chronic insomnia., 379, 1129-1141 (2012).

4. Ohayon, M. M. \& Zulley, J. Correlates of global sleep dissatisfaction in the German population., 24, 780-787 (2001).

5. Knutson, K. L. Sleep duration and cardiometabolic risk: a review of the epidemiologic evidence. Best Pract Res Clin Endocrinol Metab, 24, 731-743 (2010).

6. Knutson, K. L., Spiegel, K., Penev, P. \& Van Cauter, E. The metabolic consequences of sleep deprivation. Sleep Med Rev, 11, 163-178 (2007).

7. Festa, A., Williams, K., D'Agostino, R. Jr., Wagenknecht, L. E. \& Haffner, S. M. The natural course of beta-cell function in nondiabetic and diabetic individuals: the Insulin Resistance Atherosclerosis Study., 55, 1114-1120 (2006).

8. Han, X. et al. Long sleep duration and afternoon napping are associated with higher risk of incident diabetes in middle-aged and older Chinese: the Dongfeng-Tongji cohort study. Ann Med, 48, 216-223 (2016).

9. Yan, M. et al. Associations of sleep duration and prediabetes prevalence in a middle-aged and elderly Chinese population with regard to age and hypertension: The China Health and Retirement Longitudinal Study baseline survey. J Diabetes, 10, 847-856 (2018).

10. Ren, X. X. et al. Association between Triglyceride to HDL-C Ratio (TG/HDL-C) and Insulin Resistance in Chinese Patients with Newly Diagnosed Type 2 Diabetes Mellitus. PLoS One, 11, e0154345 (2016).

11. Zhang, Y. et al. Association of TG/HDLC ratio trajectory and risk of type 2 diabetes: A retrospective cohort study in China. J Diabetes, 13, 402-412 (2021).

12. Mokhlesi, B. et al. Association of Self-Reported Sleep and Circadian Measures With Glycemia in Adults With Prediabetes or Recently Diagnosed Untreated Type 2 Diabetes., 42, 1326-1332 (2019).

13. Shan, Z. et al. Sleep duration and risk of type 2 diabetes: a meta-analysis of prospective studies., 38 , 529-537 (2015).

14. Lu, T. Y. L. Y., Xia, P., Zhang, G. Q. \& Wu, D. R. Analysis on reliability and validity of the Pittsburgh sleep quality index. Chongqing Medicine, 43, 260-263 (2014). 
15. Krittanawong, C. et al. Association between short and long sleep durations and cardiovascular outcomes: a systematic review and meta-analysis. European Heart Journal-Acute Cardiovascular Care, 8, 762-770 (2019).

16. Tsai, P. S. et al. Psychometric evaluation of the Chinese version of the Pittsburgh Sleep Quality Index (CPSQI) in primary insomnia and control subjects. Qual Life Res, 14, 1943-1952 (2005).

17. Zheng, R. et al. Gender and age differences in the association between sleep characteristics and fasting glucose levels in Chinese adults. Diabetes Metab, 47, 101174 (2021).

18. Kim, C-R., Song, Y-M., Shin, J-Y. \& Gim, W. Association between Sleep Duration and Impaired Fasting Glucose in Korean Adults: Results from the Korean National Health and Nutrition Examination Survey 2011-2012. Korean journal of family medicine, 37, 51-56 (2016).

19. Basner, M. et al. American time use survey: Sleep time and its relationship to waking activities., 30, 1085-1095 (2007).

20. Kronholm, E., Harma, M., Hublin, C., Aro, A. R. \& Partonen, T. Self-reported sleep duration in Finnish general population. J Sleep Res, 15, 276-290 (2006).

21. Jean-Louis, G., Kripke, D. F. \& Ancoli-Israel, S. Sleep and quality of well-being., 23, 1-7 (2000).

22. Kronholm, E. et al. Trends in self-reported sleep duration and insomnia-related symptoms in Finland from 1972 to 2005: a comparative review and re-analysis of Finnish population samples. J Sleep Res, 17, 54-62 (2008).

23. Spiegel, K., Tasali, E., Penev, P. \& Van Cauter, E. Brief communication: Sleep curtailment in healthy young men is associated with decreased leptin levels, elevated ghrelin levels, and increased hunger and appetite. Ann Intern Med, 141, 846-850 (2004).

24. Rafalson, L. et al. Short Sleep Duration is Associated with the Development of Impaired Fasting Glucose: The Western New York Health Study. Ann Epidemiol, 20, 883-889 (2010).

25. Irwin, M. R., Wang, M. G., Campomayor, C. O., Collado-Hidalgo, A. \& Cole, S. Sleep deprivation and activation of morning levels of cellular and genomic markers of inflammation. Arch Intern Med, 166, 1756-1762 (2006).

26. Meier-Ewert, H. K. et al. Effect of sleep loss on C-reactive protein, an inflammatory marker of cardiovascular risk. J Am Coll Cardiol, 43, 678-683 (2004).

27. Reutrakul, S. \& Van Cauter, E. Sleep influences on obesity, insulin resistance, and risk of type 2 diabetes. Metabolism-Clinical and Experimental, 84, 56-66 (2018).

28. Tan, X., Chapman, C. D., Cedernaes, J. \& Benedict, C. Association between long sleep duration and increased risk of obesity and type 2 diabetes: A review of possible mechanisms. Sleep Med Rev, 40, 127-134 (2018).

29. Byberg, S. et al. Sleep duration and sleep quality are associated differently with alterations of glucose homeostasis. Diabet Med, 29, E354-E360 (2012).

30. Chen, P. et al. The Association Between Sleep Duration and Sleep Timing and Insulin Resistance Among Adolescents in Mexico City. The Journal of adolescent health: official publication of the 
Society for Adolescent Medicine, S1054-139X, 1-7 (2020).

31. Ford, E. S. et al. Associations between self-reported sleep duration and sleeping disorder with concentrations of fasting and 2-h glucose, insulin, and glycosylated hemoglobin among adults without diagnosed diabetes. J Diabetes, 6, 338-350 (2014).

32. Liu, J. H., Zhang, A. \& Li, L. D. Sleep duration and overweight/obesity in children: Review and implications for pediatric nursing. J Spec Pediatr Nurs, 17, 193-204 (2012).

33. Ruan, H. J., Xun, P. C., Cai, W., He, K. \& Tang, Q. Y. Habitual Sleep Duration and Risk of Childhood Obesity: Systematic Review and Dose-response Meta-analysis of Prospective Cohort Studies. Sci Rep, 5, 16160 (2015).

34. Xiao, Q., Arem, H., Moore, S. C., Hollenbeck, A. R. \& Matthews, C. E. A Large Prospective Investigation of Sleep Duration, Weight Change, and Obesity in the NIH-AARP Diet and Health Study Cohort. Am J Epidemiol, 178, 1600-1610 (2013).

35. Chaput, J. P., Despres, J. P., Bouchard, C. \& Tremblay, A. The association between sleep duration and weight gain in adults: A 6-year prospective study from the Quebec Family Study., 31, 517-523 (2008).

36. Theorell-Haglow, J., Berglund, L., Berne, C. \& Lindberg, E. Both habitual short sleepers and long sleepers are at greater risk of obesity: a population-based 10-year follow-up in women. Sleep Med, 15, 1204-1211 (2014).

37. Aziz, M. et al. Effect of Short and Long Sleep Duration in Predicting Obesity among Various Racial Groups of a Large Multi-Ethnic Organization. J Am Coll Cardiol, 71, 1850-1850 (2018).

38. Levy-Marchal, C. et al. Insulin Resistance in Children: Consensus, Perspective, and Future Directions. J Clin Endocrinol Metab, 95, 5189-5198 (2010).

39. Thumann, B. F. et al. Associations between sleep duration and insulin resistance in European children and adolescents considering the mediating role of abdominal obesity. PLoS One, 15, e0235049 (2020).

40. Statistical Bulletin of the People's Republic of China on National Economic and Social Development 2018. Available from: http://www.stats.gov.cn/tjsj/zxfb/201902/t20190228_1651265.html. Accessed 22 January 2021

41. Lockley, S. W., Skene, D. J. \& Arendt, J. Comparison between subjective and actigraphic measurement of sleep and sleep rhythms. J Sleep Res, 8, 175-183 (1999).

\section{Figures}




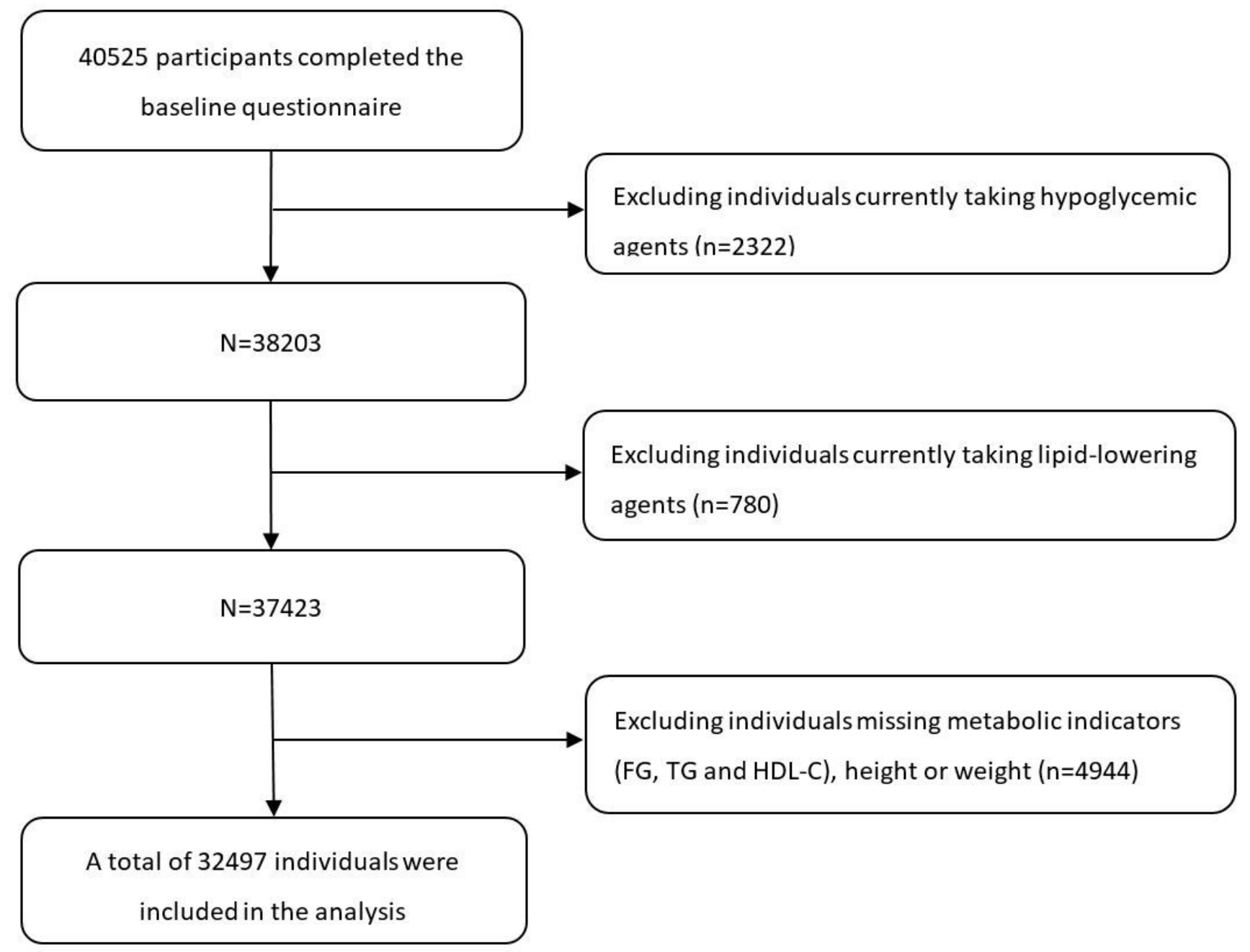

Figure 1

Flowchart of study population selection. 


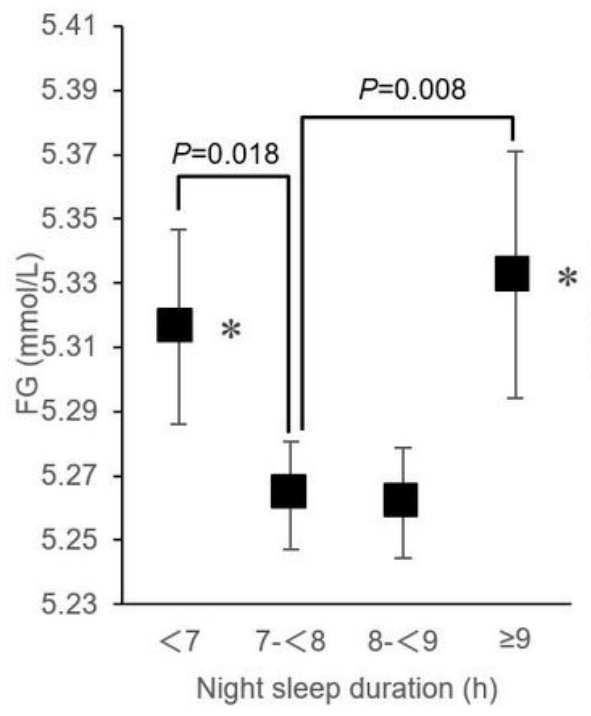

(a)

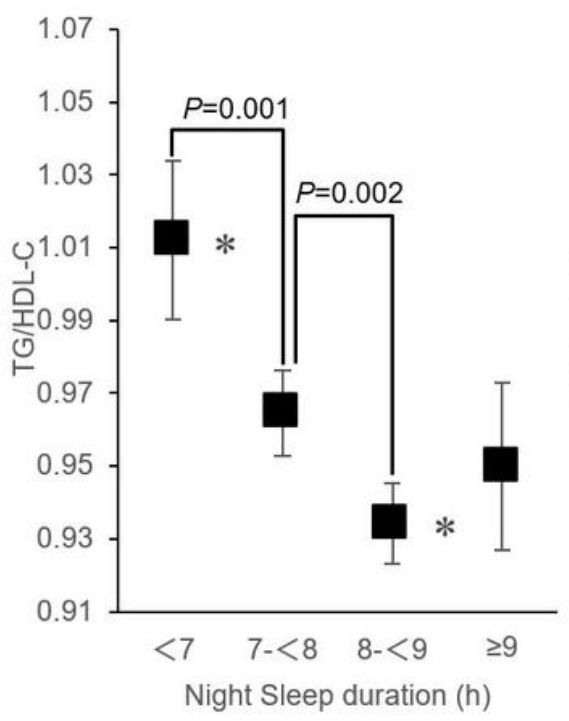

(b)

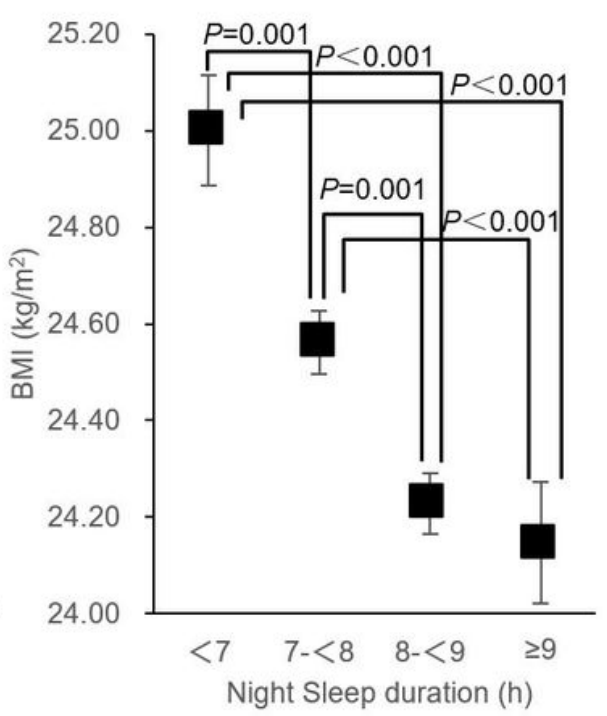

(c)

\section{Figure 2}

Mean values and $95 \%$ confidence intervals for fasting glucose, TG/HDL-C and BMI levels according to self-reported night sleep duration. TG/HDL-C is expressed as the geometric mean. * Multiple comparison results of ANOVA Pख0.05. (a) Fasting glucose concentrations in the night sleep duration $\otimes 7 \mathrm{~h}$ and $\geq 9 \mathrm{~h}$ group were significantly higher than those in the control group with night sleep duration of 7 to $<8 \mathrm{~h}$ $(\mathrm{P}=0.018, \mathrm{P}=0.008)$. (b) TG/HDL-C in the night sleep duration $₫ 7 \mathrm{~h}$ and 8 to $<9 \mathrm{~h}$ group were significantly higher than that of the control group with night sleep duration of 7 to $<8 \mathrm{~h}(\mathrm{P}=0.001, \mathrm{P}=0.002)$. (c) There were significant differences in Body mass index between the sleep duration $₫ 7 \mathrm{~h}$ group and the 7 to $<8 \mathrm{~h}, 8$ to $<9 \mathrm{~h}$ and $\geq 9 \mathrm{~h}$ groups $(P=0.001, P \otimes 0.001, P \otimes 0.001)$. There were significant differences in Body mass index between 7 to $<8 \mathrm{~h}$ group and 8 to $<9 \mathrm{~h}$ group and $\geq 9$ h group $(\mathrm{P}=0.001, \mathrm{P} \otimes 0.001)$. There was no significant difference in Body mass index between 8 to $<9 \mathrm{~h}$ group and $\geq 9 \mathrm{~h}$ group $(\mathrm{P}=0.834)$. 